\title{
Crack tip fields in ductile crystals
}

\author{
J.R. RICE, ${ }^{*}$ D.E. HAWK ${ }^{* \dagger}$ and R.J. ASARO ${ }^{\ddagger}$ \\ *Division of Applied Sciences. Harvard University, Cambridge MA 02138, USA; ${ }^{\dagger}$ Current affiliation: AT\&T Bell \\ Laboratories, Allentown, PA 18103, USA; ${ }^{+}$Department of Applied Mechanics and Engineering Sciences, \\ University of California at San Diego, La Jolla, CA 92093, USA
}

Received 1 August 1988; accepted 15 August 1988

\begin{abstract}
Results on the asymptotic analysis of crack tip fields in elastic-plastic single crystals are presented and some preliminary results of finite element solutions for cracked solids of this type are summarized. In the cases studied, involving plane strain tensile and anti-plane shear cracks in ideally plastic $f \mathrm{cc}$ and bcccrystals, analyzed within conventional small displacement gradient assumptions, the asymptotic analyses reveal striking discontinuous fields at the crack tip.

For the stationary crack the stress state is found to be locally uniform in each of a family of angular sectors at the crack tip, but to jump discontinuously at sector boundaries, which are also the surfaces of shear discontinuities in the displacement field. For the quasi-statically growing crack the stress state is fully continuous from one near-tip angular sector to the next, but now some of the sectors involve elastic unloading from, and reloading to. a yielded state, and shear discontinuities of the velocity field develop at sector boundaries. In an anti-plane case studied, inclusion of inertial terms for (dynamically) growing cracks restores a discontinuous stress field at the tip which moves through the material as an elastic-plastic shock wave. For high symmetry crack orientations relative to the crystal, the discontinuity surfaces are sometimes coincident with the active crystal slip planes, but as often lie perpendicular to the family of active slip planes so that the discontinuities correspond to a kinking mode of shear.

The finite element studies so far attempted, simulating the ideally plastic material model in a small displacement gradient type program, appear to be consistent with the asymptotic analyses. Small scale yielding solutions confirm the expected discontinuities, within limits of mesh resolution, of displacement for a stationary crack and of velocity for quasi-static growth. Further, the discontinuities apparently extend well into the near-tip plastic zone. A finite element formulation suitable for arbitrary deformation has been used to solve for the plane strain tension of a Taylor-hardening crystal panel containing, a center crack with an initially rounded tip. This shows effects due to lattice rotation, which distinguishes the regular versus kinking shear modes of crack tip relaxation. and holds promise for exploring the mechanics of crack opening at the tip.
\end{abstract}

\section{Introduction}

This paper summarizes recent analytical and numerical investigations into the nature of the near-crack-tip stress and deformation fields in ductile single crystals. Ductile crystals deform plastically by the motion of dislocations on a limited set of slip systems. A continuum representation of this plastic deformation consistent with the Schmid rule, which states that flow on a system is activated when the shear stress resolved on that system reaches a critical value, is used in the analyses to be presented. This formulation leads to a yield surface in stress space consisting of planar facets joined at vertices and to an "associated" plastic straining relation.

General methods of constructing asymptotic near-tip fields for such crystals, with either stationary or quasi-statically growing cracks, have been obtained in the ideally plastic case for both anti-plane strain (mode III: [1]) and tensile plane strain (mode I: [2]) cracks. The results, as illustrated for common crack orientations in $\mathrm{fcc}$ and bcc crystals, lead to 
striking predictions of discontinuities at the crack tip. Full scale elastic-ideally plastic solutions to the near-tip stress and deformation fields have been given for stationary cracks in the mode III study. These show that all flow is confined to planar plastic zones emanating from the crack tip, across which both displacement and stress are discontinuous. Asymptotic analysis of dynamic crack growth, i.e., including inertia, has been developed as well for that mode [3] and reveals that an elastic-plastic shock discontinuity moves along with the tip. In addition, asymptotic fields of the HRR type have been developed for stationary cracks in crystals showing Taylor hardening, with a power-law stress-strain relation at large strain, in mode III [4] and mode I [5].

The mode I asymptotic analysis [2], based on ideal plasticity and a "small displacement gradient" formulation, shows that for material at yield the stress state is constant within angular sectors whose boundaries are certain crystallographic directions on which discontinuities in either displacement (stationary crack) or velocity (quasi-statically growing crack) are possible. A direct comparison of the mode I analyses is made here with the numerical results of Hawk and Asaro [6]. When comparing different types of crystals, or crack orientations within a given crystal, the structure of the dislocations necessary to produce the same continuum field discontinuity is different. Furthermore, certain dislocations structures may induce rotation of the lattice relative to the material, thus changing the resolved shear stresses on slip systems and causing a geometric hardening or softening of the crystal. This is important particularly when large deformations are taken into account.

Recently, full scale elastic-plastic solutions for mode I cracks in ductile crystals have been obtained numerically by the detailed finite element analyses of Hawk and Asaro [6]. Some of their results are briefly summarized here for several different loading cases. These analyses model the constitutive behavior of the crystal with a visco-plastic formulation in the nearly rate-independent limit. The numerical analysis of Asaro [6] models from small-scale to general yielding a center cracked panel with a blunted crack tip under uni-axial tension. The slip systems of the crystal are idealized by a planar double-slip model. The effect of Taylor type hardening of the slip systems and large displacement gradients (e.g., lattice rotation) are included. In the small-scale-yielding analysis of Hawk [6], both a stationary and quasistatically propagating perfectly sharp tensile crack are simulated. The crystal is modeled as elastic-ideally plastic using a complete description of the slip systems in a f c c crystal. Small displacement gradients are assumed.

\section{Constitutive law}

The crystals considered can undergo both elastic and plastic deformation. The plastic deformation is consistent with a continuum description of single crystals [7-10]. The total strain-rate is taken as the sum of the elastic strain-rate and plastic strain-rate

$$
\dot{\varepsilon}_{i j}=\dot{\varepsilon}_{i j}^{e}+\dot{\varepsilon}_{i j}^{p}
$$

Under plane strain conditions, which are possible for the high-symmetry crack orientations considered here, $\dot{\varepsilon}_{13}, \dot{\varepsilon}_{23}, \dot{\varepsilon}_{33}, \sigma_{13}$ and $\sigma_{23}$ are zero. The plastic deformation of the crystal occurs by the motion of dislocations along certain preferential crystallographic planes. The movement of these dislocations causes a permanent dilationless straining of the crystal. A slip 
system denoted by $\alpha$ is defined by two unit vectors giving the slip direction $\mathbf{s}^{(x)}$ and the normal to the slip plane $\mathbf{n}^{(x)}$. The parameter $\gamma^{(x)}$ describes the amount of shear strain on each slip system. These preferential slip systems defined by $\mathbf{s}^{(x)}$ and $\mathbf{n}^{(x)}$ vary according to the crystal structure (e.g., face centered cubic, f c c, or body centered cubic, b cc). It is possible to express the plastic strain rate as a sum over all the $N$ slip systems of the crystal as

$$
\dot{\varepsilon}_{i j}^{p}=\sum_{\alpha=1}^{N} \mu_{i j}^{(\alpha)} \dot{\gamma}^{(\alpha)}
$$

Here $\mu_{i j}^{(\alpha)}$ is termed the Schmid factor and is determined from the slip direction $\mathbf{s}^{(\alpha)}$ and the normal to the slip plane $\mathbf{n}^{(x)}$ as

$$
\mu_{i j}^{(\alpha)}=\frac{1}{2}\left(s_{i}^{(\alpha)} n_{j}^{(\alpha)}+s_{j}^{(\alpha)} n_{i}^{(\alpha)}\right)
$$

The resolved shear stress, $\tau^{(x)}$, on a system is expressible in terms of these Schmid factors as

$$
\tau^{(\alpha)}=\sigma_{i j} \mu_{i j}^{(\alpha)}
$$

The plastic deformation of a crystal is said to obey the Schmid rule if the instantaneous shear response $\dot{\gamma}^{(x)}$ of any given system $\alpha$ depends on the current stress state only through the combination in (4). In a time-independent plasticity formulation, a necessary but not sufficient condition for slip to occur is that the resolved shear stress $\tau^{(x)}$ reaches a critical value $g^{(z)}$. This criteria results in a yield surface in stress space which consists of planar facets that join at vertices at which two or more systems are simultaneously active. Equation (2) then assures that the flow rule is of an "associated" type. Rice [11] showed that for rigid-plastic incompressible solids of this class in plane flow, the yield surface can be represented as a curve in the reduced stress space of $\left(\sigma_{11}-\sigma_{22}\right) / 2$ and $\sigma_{12}$. Such a yield surface is schematically shown in Fig. 1 and for crystals as described here, it is polygonal. In an elastic-plastic

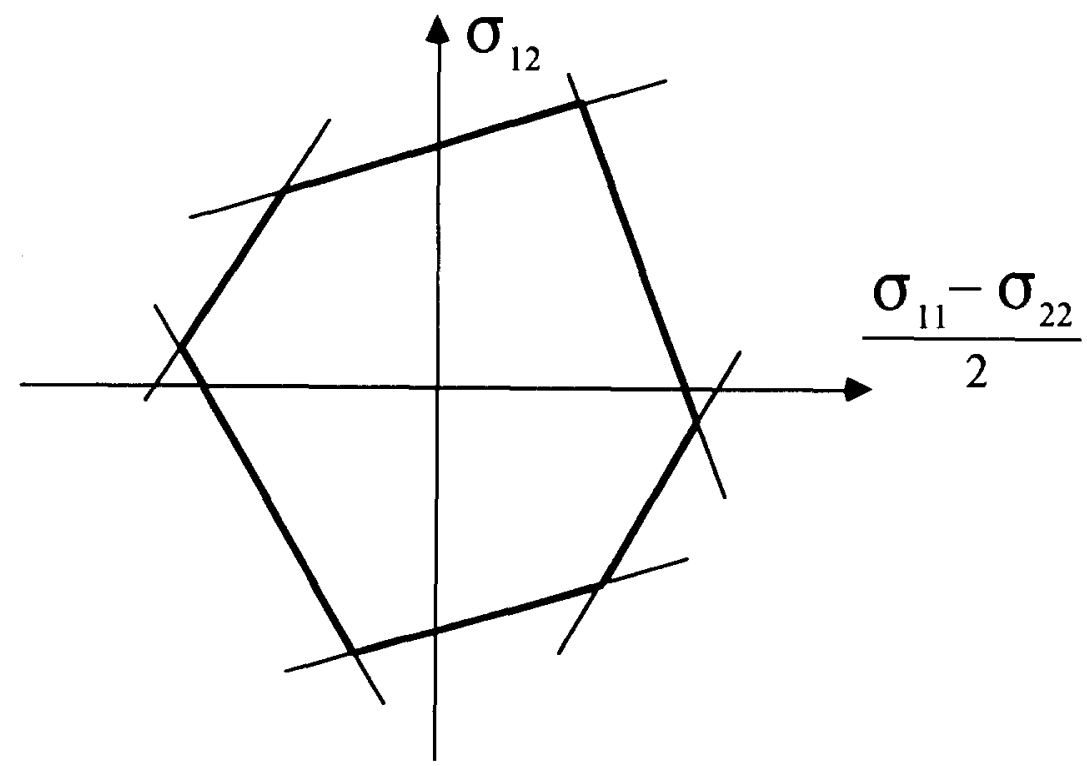

Fig. I. Schematic of yield surface for a ductile crystal undergoing plane straining. 
material the same result applies for stress states in large sustained plastic strain. However, in such a case limited deformation may occur on slip systems corresponding to segments of the yield surface sensitive to the value of $\left(\sigma_{11}+\sigma_{22}\right)$ but which cannot produce sustained plane flow. We will term the sides of the polygon as "flats" and the point of junction of two sides as a "vertex". The flow law of (2) being associative, the direction of plastic straining, with components $\left(\dot{\varepsilon}_{11}^{p}-\dot{\varepsilon}_{11}^{p}\right) / 2$ and $\dot{\varepsilon}_{12}^{p}$, is normal to the yield surface on a "flat" and within the forward fan of normals at a "vertex".

It will also be convenient to represent the slip systems of the crystal as traces in the $x_{1}, x_{2}$ plane. For example, in Fig. 2a, a unit cube representing the lattice of an $\mathrm{fcc}$ crystal is shown. The slip planes of a $\mathrm{f} \mathrm{c}$ crystal are the $\left\{\begin{array}{lll}1 & 1 & 1\end{array}\right\}$ planes indicated by the various shaded planes in the cube. The slip directions are $\langle 101\rangle$ directions which are the diagonals of the cube faces. If the crack lies in the plane $(010)$ and the crack tip lies along the diagonal of the cube face as indicated, then the traces of the slip systems (i.e. intersection of the slip planes with the $x_{1}, x_{2}$ plane) are as indicated in Fig. 2b. It is then possible to represent the slip direction $\mathbf{s}$ and slip plane normal $\mathbf{n}$ as the directions $\mathbf{S}$ and $\mathbf{N}$ respectively in the $x_{1}, x_{2}$ plane as shown in the figure. Only those traces shown correspond to systems which can produce sustained

(a)

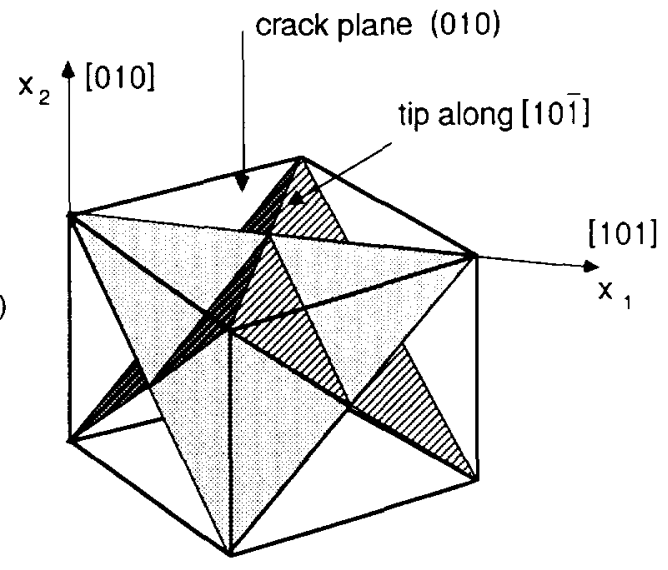

(b)

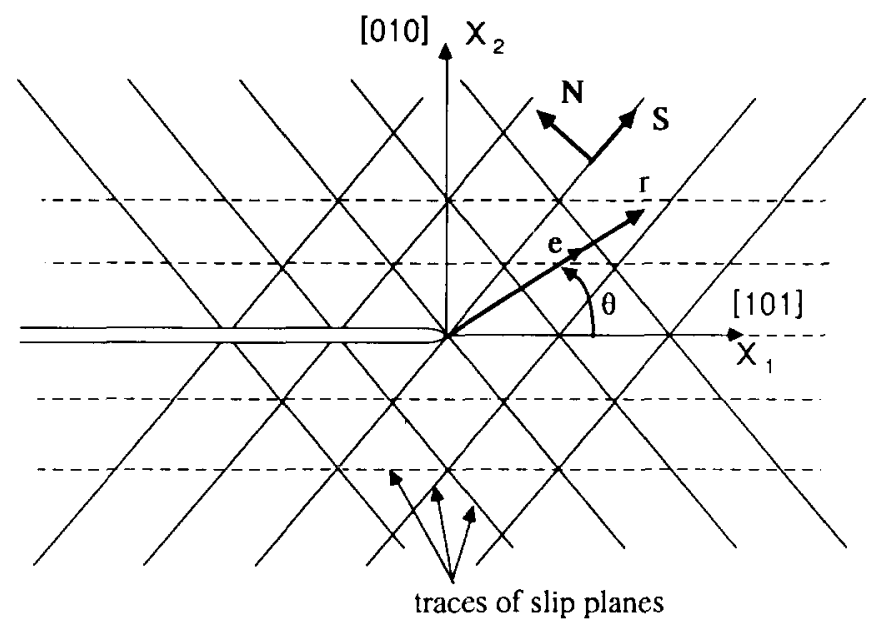

Fig. 2. (a) Face centered cubic slip systems, and orientation of the crack plane and crack tip for case illustrated below. (b) Crack on $\left(\begin{array}{lll}0 & 1 & 0\end{array}\right)$ plane growing in [ $\left.\begin{array}{llll}1 & 0 & 1\end{array}\right]$ direction. Traces are shown of those slip plane families which can accommodate sustained plane straining. 
plane flow. The solid line traces correspond to individual $\left\{\begin{array}{lll}1 & 1\end{array}\right\}$ planes, and the dashed lines to two such planes which intersect along a $\langle 110\rangle$ slip direction common for each and which, by equal coincident slip, produce plane flow.

The finite element analyses use a visco-plastic formulation where slip can occur on any system so long as resolved shear stress on that system is non-zero. A simple power-law relation [12-14] can be used to describe the rate of slip on each system as

$$
\dot{\gamma}^{(\alpha)}=\dot{\gamma}_{0}^{(\alpha)} \operatorname{sgn}\left(\tau^{(\alpha)}\right)\left(\left|\frac{\tau^{(x)}}{g^{(\alpha)}}\right|\right)^{1 / m}
$$

As the exponent $m \rightarrow 0$ the visco-plastic model approaches the rate-independent formulation. Use of a visco-plastic law in a finite element formulation, pioneered in the work of Pierce et al. [14], eliminates certain problems of uniqueness and considerations of when a point is at yield. The $g^{(x)}$ reflects the current level of strain hardening in the crystal. In [6], results are presented for both ideal plasticity i.e., $g^{(x)}$ is a constant, $\tau_{0}$, and cases where $g^{(\alpha)}$ is a function of the sum of the magnitudes of the slips $\left(\Gamma=\Sigma_{x}\left|\gamma^{(x)}\right|\right)$, which coincides with Taylor hardening.

\section{Asymptotic analysis}

The asymptotic analyses (e.g., $\lim _{r \rightarrow 0} \sigma_{\alpha \beta}(r, \theta, t)$ ) of Rice [2] are summarized here for the case of ideal plasticity in a "small displacement gradient" formulation of the mode I problem. Based on the equations of equilibrium with a bounded crack tip stress state and with the further condition that the stress state is at yield relative to a slip plane trace in the direction $S_{j}$, the following requirement is found:

$$
\left(N_{\alpha} e_{\alpha}\right)\left(S_{i} e_{\lambda}\right)\left(\sigma_{11}^{\prime}+\sigma_{22}^{\prime}\right)=0
$$

Greek indices range from 1 to 2 . As indicated, Fig. 2b, the $e_{\alpha}$ are the components of the radial unit vector in the $x_{1}, x_{2}$ plane; $\left(e_{1}, e_{2}\right)=(\cos \theta, \sin \theta)$. The terms $S_{\alpha}$ and $N_{\alpha}$ are the components of the traces of the slip direction and slip plane normal as previously defined. The $\sigma_{\alpha \beta}^{\prime}$ denote

$$
\sigma_{x \beta}^{\prime}=\lim _{r \rightarrow 0} \frac{\partial \sigma_{x \beta}(r, \theta)}{\partial \theta}
$$

Equation (6) implies $\sigma_{11}^{\prime}+\sigma_{22}^{\prime}=0$ for all $\theta$ except for four special values when e is aligned with either $\mathbf{N}$ or $\mathbf{S}$. Based on the form of the stresses consistent with equilibrium for material at yield, this statement further implies that within sectors bounded by these four special values of $\theta$, all $\sigma_{x \beta}^{\prime}$ are zero; i.e. the stress state is independent of $\theta$. For a tensile crack it is shown that, in order to meet boundary conditions, either (i) the stresses in certain angular sectors around the crack tip are not at yield or (ii) that the stresses change discontinuously at the special values of $\theta$ as mentioned above. 


\subsection{Stationary crack}

For the stationary crack, as $r \rightarrow 0$, the yield condition can be met in all angular sectors around the crack tip, thus requiring discontinuous jumps in the stress state. The stress discontinuity must be from one point on the yield surface to another, and considering the nature of restrictions on such discontinuities, this path must be a straight line in the stress space and the line must lie everywhere along the yield locus (and hence correspond to a flat segment in Fig. 1) since otherwise elastic unloading would occur. The solution which satisfies the jump conditions for the stress discontinuity is one where the stress state changes from vertex to vertex on the yield surface. The deformation fields, consistent with the above stress state, must have a shear type of discontinuity in the displacement field along the same direction, e, for which the stress is discontinuous. These correspond to concentrated shear parallel to the slip plane traces when $\mathbf{e}$ is aligned with $\mathbf{S}$, and to kink-like shear perpendicular to the traces when $\mathbf{e}$ is aligned with $\mathbf{N}$. The specifics of constructing such solutions may be found in [2]. Specific examples of these solutions will be given in the discussion of the finite element solutions of [6].

\subsection{Growing crack}

For the quasi-statically growing crack, Drugan and Rice [15] demonstrated for this type of material that discontinuities in stresses and displacements cannot exist. However, it is possible to have velocity discontinuities. It is therefore necessary to have bordering on the plastic sectors angular sectors in which elastic unloading (and perhaps reloading) occur. Construction of the solution for a particular crystal orientation, again for which the details are given in [2], shows a complicated pattern of plastic sectors and elastic unloading and reloading sectors is necessary to model the crack tip fields for quasi-static growth. A specific example will be presented for comparison with the finite element solutions of Hawk [6] in the next section which shows that one of the physically active system for the stationary crack becomes inactive in the growing case.

\subsection{Note on role of $\boldsymbol{S}$ and $\boldsymbol{N}$}

In the asymptotic analyses presented, based on the "small displacement gradient" formulation, the role of the slip direction $\mathbf{S}$ and slip plane normal $\mathbf{N}$ are interchangeable since they come in through the symmetric form of (3). In certain cases [2] f c c crystals and b c c crystals differ only by an interchange of $\mathbf{S}$ and $\mathbf{N}$. Therefore solutions obtained for such $\mathrm{fcc}$ crystals are also valid for b c c crystals as well (aside from a slight scaling). However, the structure of the actual dislocations necessary to produce the shear deformation is quite different when $\mathbf{S}$ and $\mathbf{N}$ are reversed. For example, shown in Fig. $3 \mathrm{a}$ and $3 \mathrm{~b}$ is shear parallel to the slip planes and shear perpendicular to the slip planes respectively. The motion indicated in Fig. 3a can be generated by the emission of dislocations from the crack tip along the slip direction as shown in Fig. 3c. Notice however that in Figure 3b the slip planes must form a kink in order to accommodate the deformation. Experiments in $\mathrm{Fe}-3 \% \mathrm{Si}$ crystals [16] show results consistent with kink-like shear. This kink requires dislocation dipoles as in Fig. 3d. These dipole loops, illustrated as pairs of dislocations, cannot be swept out from the crack tip and 

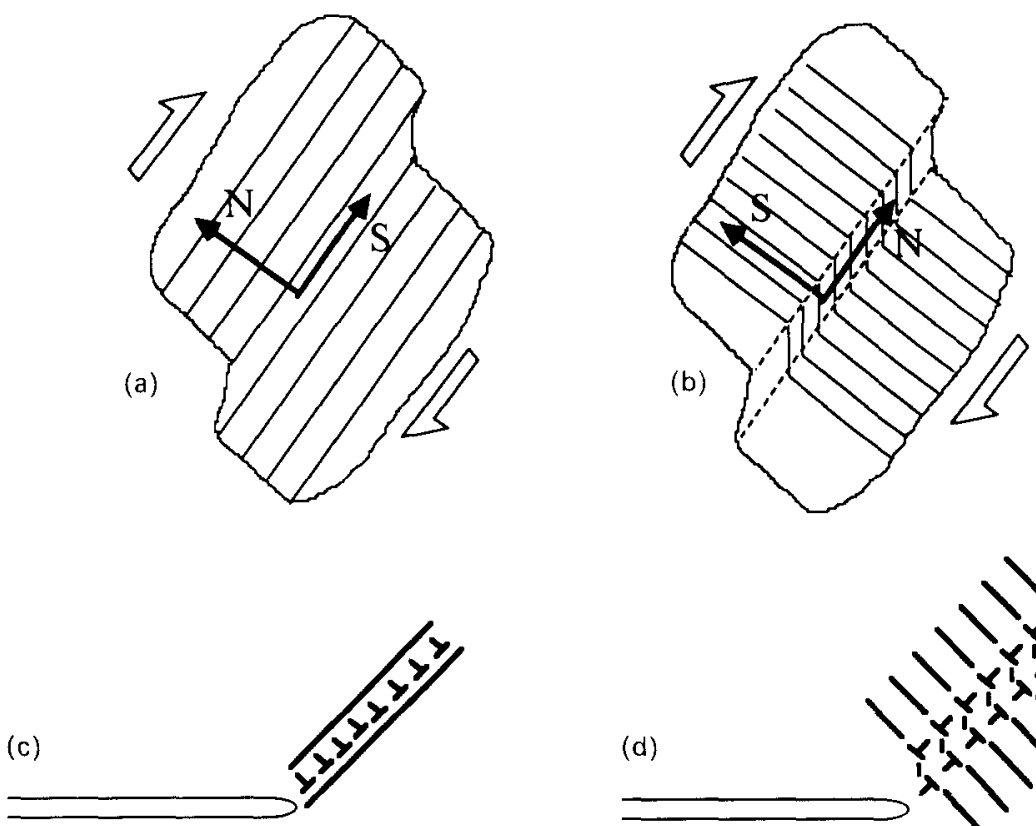

(d)

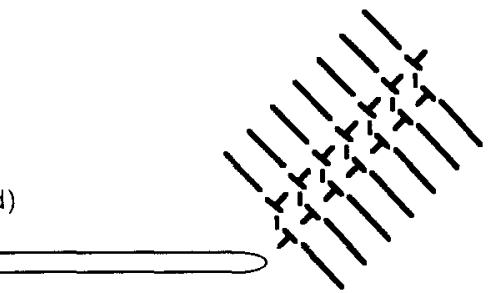

Fig. 3. (a) Shear in band lying parallel to the active slip systems. (b) Shear by a kinking mode in a band lying perpendicular to the active system. (c) Dislocations can be generated at the crack tip and swept out along slip planes, or can be generated from internal sources, to produce the slip-plane-parallel shear band of (a). (d) Dislocations dipole loops must be nucleated from internal sources and expand as illustrated to produce the kinking shear band of (b).

must therefore depend on the availability of internal sources to produce them. Requiring the availability of internal sources, as opposed to allowing dislocations to be swept out from the crack tip along slip planes, may influence whether the fracture of a particular crystal is brittle or ductile. It is seen also that the lattice between the pair of dislocations in Fig. $3 \mathrm{~d}$ is apparently rotated. This can cause either a geometric hardening or softening of the material because the resolved shear stress $\tau^{(\alpha)}$ on each slip system varies as the Schmid factor, $\mu_{i j}^{(\alpha)}$, changes due to rotation of $\mathbf{S}$ and $\mathbf{N}$ directions. The effect of such lattice rotation will be seen later in the large deformation results of Asaro [6].

\subsection{Some further analyses of mode III cracks}

The analyses discussed so far have been for tensile cracks, which is the physically more interesting case. However they are approximate in that their validity is only in the limit as $r \rightarrow 0$. The simpler nature of the equations for mode III (anti-plane strain) type loading allows for more complete solutions [1,3] which we briefly summarize here. Three such solutions are shown in Fig. 4 exclusively for the f c crystal with the orientation of the crack the same as in Fig. 2a with the crack along the $(010)$ plane with crack tip along the $\left[\begin{array}{lll}1 & 0 & \overline{1}\end{array}\right]$ direction. Under mode III loading conditions the only non-zero displacement is $u_{3}\left(x_{1}, x_{2}\right)$ which gives rise to only two non-zero stress components $\sigma_{31}$ and $\sigma_{32}$. In this case only the solid-line slip plane traces in Fig. $2 \mathrm{~b}$ can provide anti-plane straining. The yield surface can thus be represented in $\sigma_{31}, \sigma_{32}$ stress space and for this orientation is diamond 
(a)

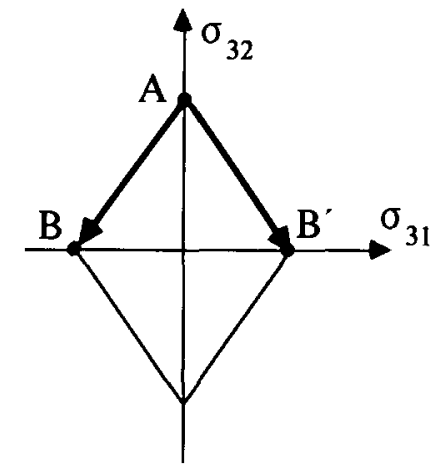

(b)
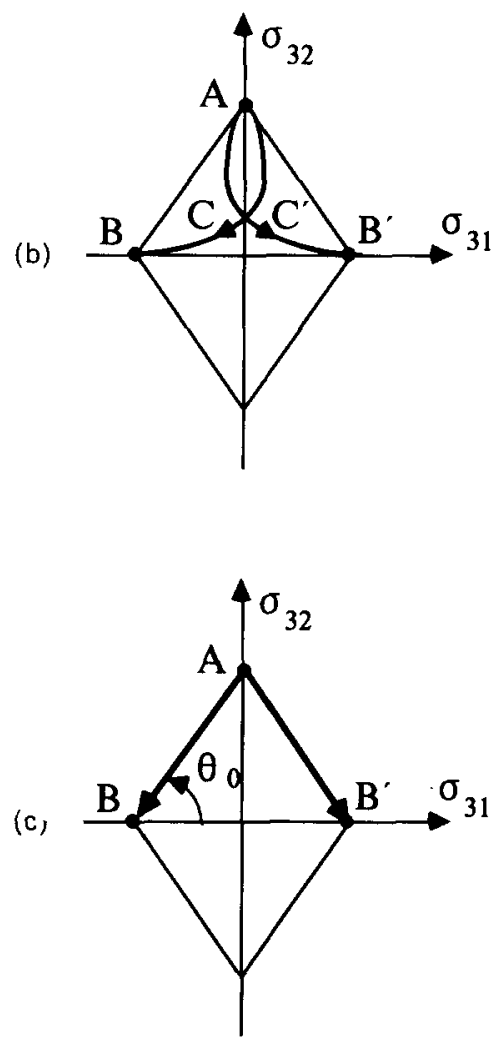
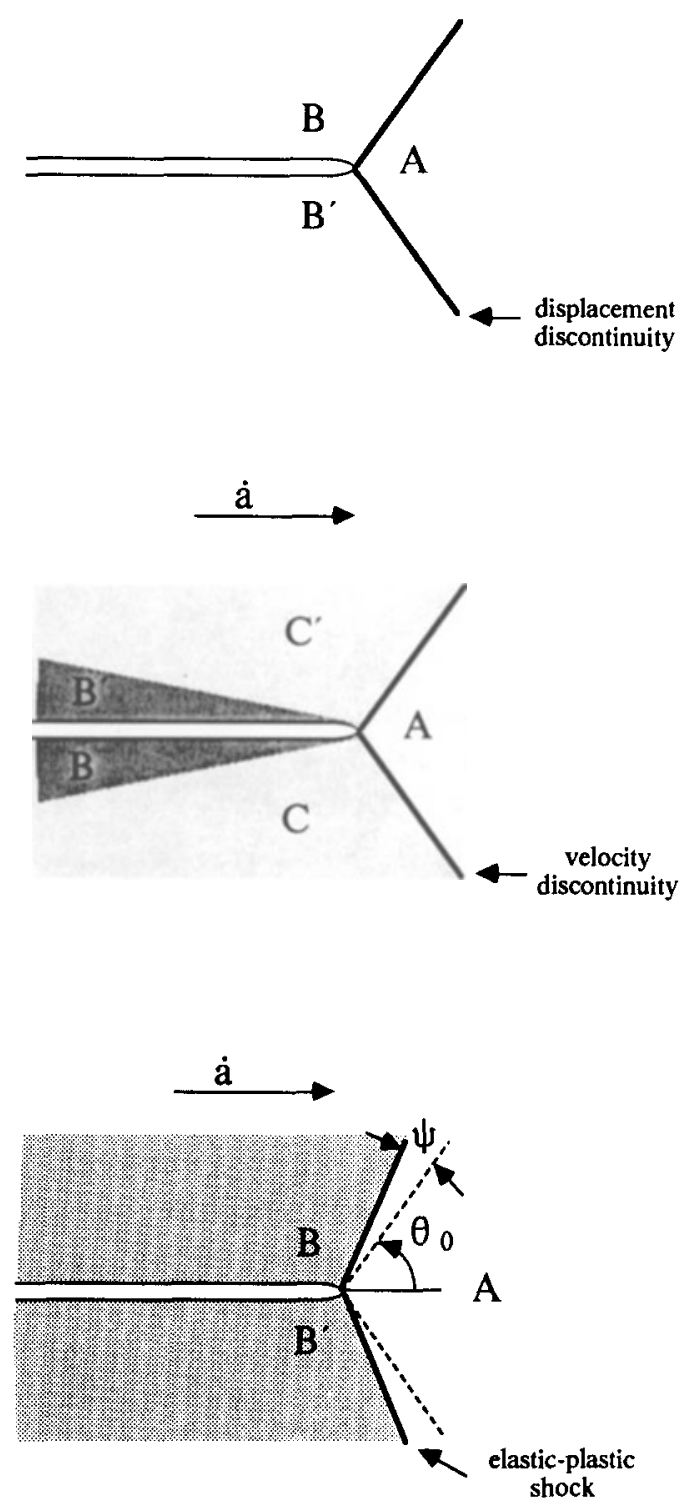

Fig. 4. Summary of analyses for anti-plane shear of a cracked ideally plastic crystal: (a) Stationary crack; (b) Quasi-static crack growth; (c) Dynamic crack growth asymptotic field (inertial effects included).

shaped. The assembled stress sectors for these solutions will be labeled with $A, B$, etc. with corresponding points or trajectories labeled on the yield surface. As is often the case, similar features are seen to carry over from the mode III solution to the mode I solution.

The stationary case is shown in Fig. 4a with the corresponding yield surface and stress trajectories as indicated. Only two sectors $A, B$ in the upper half plane (a corresponding sector to $B$ labeled $\mathbf{B}^{\prime}$ is shown in the lower half plane) of the constant stress type, as previously discussed for plane strain in the asymptotic limit as $r \rightarrow 0$, are necessary to construct the full solution. Both of the corresponding stress points are on the yield surface 
as indicated. These sectors are separated by a line of discontinuity in both displacement and stress. The stress discontinuity can be thought of as a rapid transition (in a vanishing small sector) from point $A$ to point $B$ as indicated by the arrow on the yield surface. The complete solution of Rice and Nikolic [1] shows that these sectors are actually elastic with all plasticity collapsed into a shear zone along the line of the displacement discontinuity. The size of this plastic zone is found exactly in their analysis.

The quasi-static crack growth case is shown in Fig. 4b. Three sectors $A, B^{\prime}$ and $C^{\prime}$ in the upper half plane (with corresponding sectors $B$ and $C$ in the lower half plane) are necessary to assemble the solution. Sector $A$ is of the constant stress type with the state of stress at the yield surface as indicated on the yield surface plot. As necessary from the asymptotic solution this sector is bounded by an elastic sector $C^{\prime}$. Sector $A$ is separated from $C^{\prime}$ by a velocity discontinuity (expected to extend over a finite size region based on the approximate analysis of Rice and Nikolic [1]) along which all the plastic flow takes place in the region forward of the crack tip. As shown by the lightly shaded region in Fig. 4b, as the crack grows this velocity discontinuity also leaves behind a wake of plastic deformation. Sector $C^{\prime}$, as indicated by the corresponding path within the yield surface, unloads elastically and reloads to yield by the time it reaches the other plastic sector $B^{\prime}$ creating a thin wedge of plastic deformation along the crack face, shown in darker shading. The exact details of the construction of the angles for these sectors is presented in [1].

Finally, the case of dynamic crack growth where the effects of inertia are taken into account is shown in Fig. 4c [3]. In this case the entire field consists of constant stress sectors separated by an elastic-plastic shock (possible only in single-crystal-like materials with flat segments along their yield locus). Both velocity and stress are discontinuous across the shock. Remarkably, the strain accumulated in crossing the shock is finite at the crack tip. It is of the order of the strain at first yield divided by the elastic Mach number associated with the crack speed. The angle $\psi$, in Fig. 4c, is proportional to the Mach number at low speed and approaches $\left(\pi-\theta_{0}\right) / 2$ at the sonic speed. There must be a complicated transition between the near tip field with the inertia included, Fig. 4c, and that without Fig. 4b. This is an example of nonuniform asymptotic limits as one considers $r \rightarrow 0$ and $\sqrt{\varrho v^{2} / G} \rightarrow 0$ where $\varrho$ is the density, $G$ is the shear modulus and $v$ is the crack speed. E.g., the quasi-static growth case may be considered as letting $\varrho \rightarrow 0$, or $v \rightarrow 0^{+}$, before letting $r \rightarrow 0$.

\section{Comparison of asymptotic and numerical analyses for mode I}

\subsection{Small-scale-yielding results}

Under small-scale-yielding conditions, a stationary and quasi-statically propagating plane strain mode I crack in a f c c crystal has been investigated by the finite element analysis of Hawk [6]. The material is elastic-ideally plastic, modeled by a visco-plastic formulation as in (5), with $g^{(x)}=\tau_{0}$ and $m=0.005$. Small-scale-yielding conditions exist when the size of the plastic zone is much smaller than the region over which the elastic singular stress field dominates. The dominance of the elastic singularity allows the crack tip region to be modeled as an infinite solid with a semi-infinite crack where the stresses approach those of the elastic singularity as $r \rightarrow \infty$. The finite element mesh of both analyses is shown in Fig. 5. Since the mode I problem is symmetric, only the upper half plane is modeled. The finite element mesh 

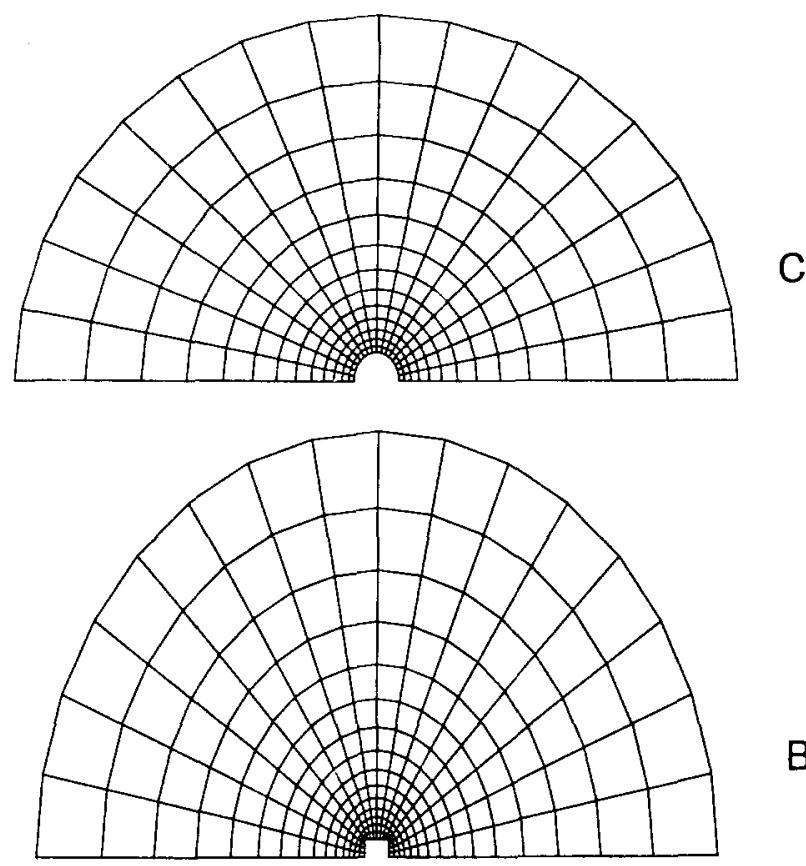

B

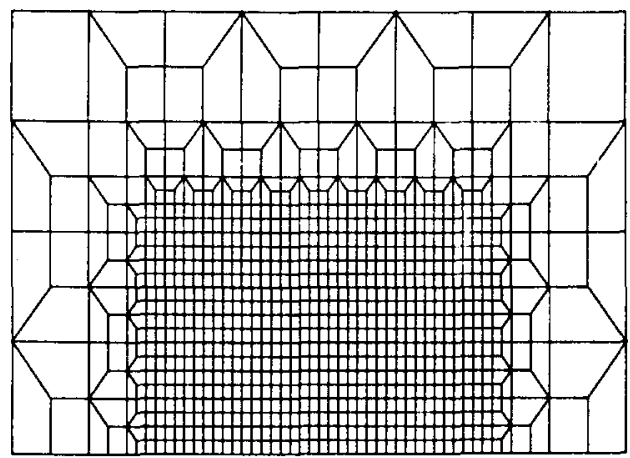

Fig. 5. Finite element mesh for "small displacement gradient" analysis of small-scale yielding in an ideally plastic crystal.

is shown in three sections $\mathrm{A}, \mathrm{B}$ and $\mathrm{C}$ for clarity. Section $\mathrm{A}$ fits into the rectangular space of section $\mathrm{B}$ and section $\mathrm{B}$ in turn fits into the space in section $\mathrm{C}$. The ratio of the size of the smallest rectangular element of region $\mathrm{A}$ to the outermost radius of region $\mathrm{C}$ is approximately $10^{-4}$. Each quadrilateral element is actually a so called cross triangle element [17] made up of four constant-strain triangles (formed from the diagonals of the element) which as a group behave well under incompressible conditions. The crack opens to the left with nodes ahead of the crack tip constrained from vertical movement by the symmetry boundary condition. Tractions corresponding to the elastic $\mathrm{K}$ field singularity are applied to the outer boundary of section $\mathrm{C}$. The results are primarily from section $\mathrm{A}$ and the region immediately surrounding it. The details of the finite element method are presented in [6].

The analysis of the stationary crack in an $f c c$ crystal was performed with the orientation of the crack the same as shown in Fig. 2a. The crack lies in the (010) plane with the crack tip along the $\left[\begin{array}{lll}1 & 0 & \overline{1}\end{array}\right]$ direction. Elasticity was modeled as isotropic. The yield surface 


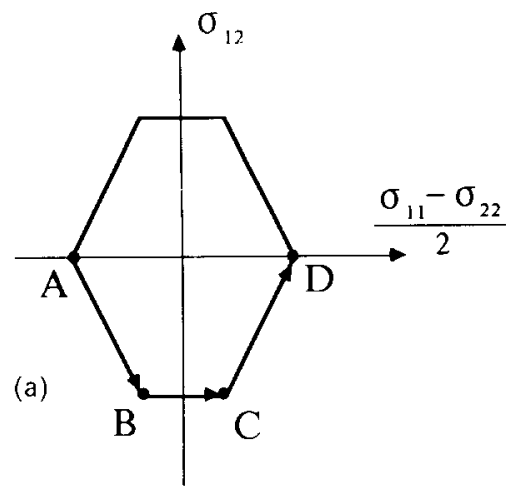

(b)

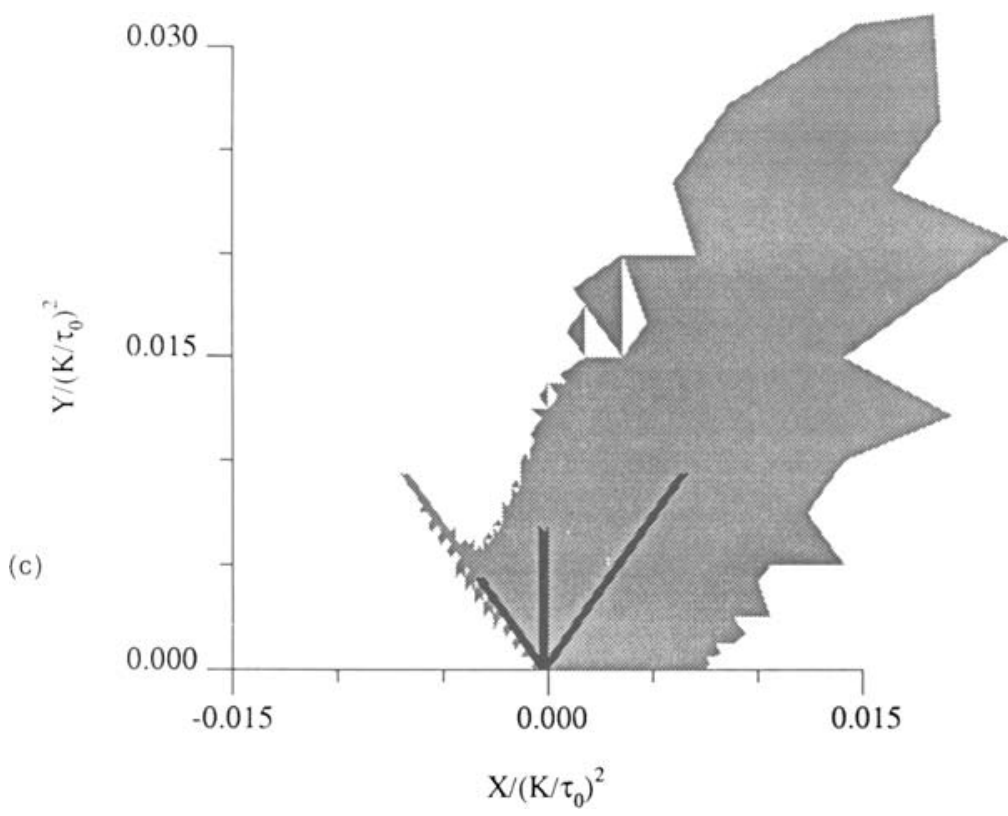

Fig. 6. Stationary crack in ideally plastic crystal of Fig. 2: (a) Yield surface; (b) Asymptotic structure of crack tip field; (c) Finite element results: grey zone with $\Gamma \geqslant 0.01\left(\tau_{0} / G\right)$ essentially denotes plastic zone; black zone with $\Gamma \geqslant 10\left(\tau_{0} / G\right)$ shows zone of more concentrated plastic strain.

(appropriate to sustained flow) for this orientation is shown in Fig. 6a. The asymptotic solution obtained by Rice [2] for the stationary crack case is shown in Fig. 6b. In the stationary case, the entire crack tip region may deform plastically. For the upper half plane, the solution consists of four constant stress sectors labeled A, B, C, and D separated by stress and displacement discontinuities as indicated in Fig. 6b. The stress state of each sector is similarly labeled as points on the yield surface A, B, C and D in Fig. 6a. The discontinuity between $\mathrm{A}$ and $\mathrm{B}$ at $54.7^{\circ}$ and the one between $\mathrm{C}$ and $\mathrm{D}$ at $125.3^{\circ}$ correspond to the slip directions for the solid traces in Fig. 2b. The discontinuity between sectors B and C corresponds to the normal of the slip plane indicated by the dashed traces in Fig. 2b. Each discontinuity in stress represents a rapid transition from point to point on the yield surface as shown by the arrows.

Let the sum over the slip systems of the magnitude of slip, $\Sigma_{\alpha}\left|\gamma^{(x)}\right|$ be denoted as $\Gamma$. This quantity is an overall measure of the amount of plastic straining and is shown in Fig. $6 \mathrm{c}$ 
normalized by the elastic strain at yield, $\tau_{0} / G$. The grey zone, formed by shading individual triangular elements whose value of $\Gamma /\left(\tau_{0} / G\right)$ is between 0.01 and 10 , is representative of the overall plastic zone shape. The black bands are those elements whose value of $\Gamma$ is greater than 10. Therefore, the most intense straining is along the predicted discontinuities of the asymptotic solution. It is interesting to note that all the sectors are predicted to be stressed to yield as $r \rightarrow 0$, but only the interfaces between them are proven to be deforming plastically in the asymptotics. The finite element solution indicates that sector D bounding the crack face is relatively free of plastic deformation while the others deform. Some plastic strain

(a)
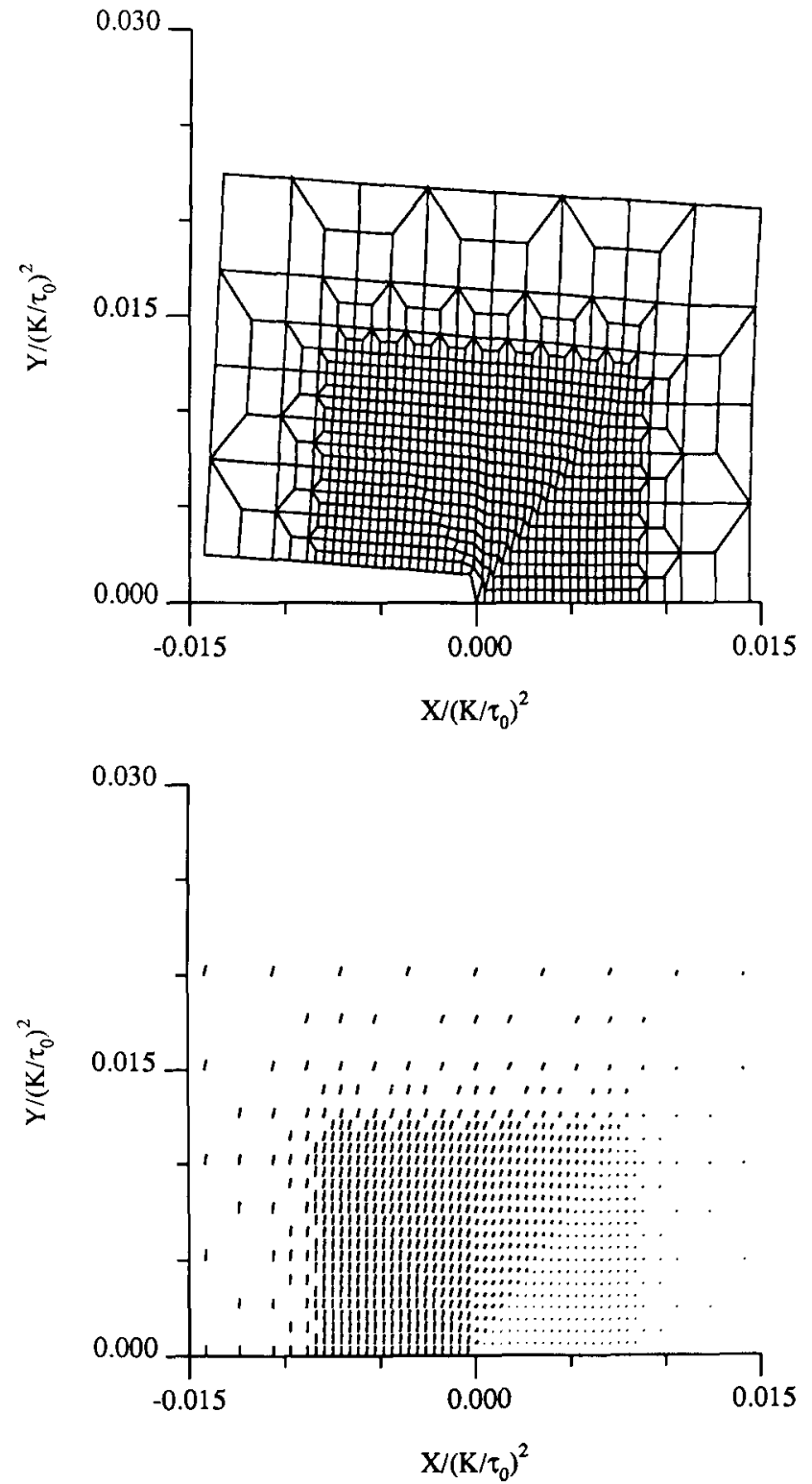

Fig. 7. (a) Deformed mesh for stationary crack (factor of 50 amplification of displacements); note shear zones coincident with directions shown in Fig. 6(b). (b) Line lengths proportional to displacements accumulated in a load increment; suggests that displacement discontinuities predicted by asymptotic analysis extend well into plastic zone. 
ahead of the crack occurs on slip systems other than those of Fig. 2b, i.e., on systems which cannot undergo sustained plane flow. The deformation of the finite element mesh around the crack tip is shown in Fig. 7a with the displacements magnified by a factor of 50 . The most intense deformation is in those elements which lie along the discontinuity between sectors $\mathrm{A}$ and B. The increment of displacement, scaled up for visibility, over a load increment is shown in Fig. 7b. Four distinct sectors exist with the motion in each sector fairly uniform. This indicates the majority of deformation is caused by the movement of nodes near the predicted discontinuities.

A quasi-statically propagating crack in a fcc crystal is simulated using a node release technique the details of which are given in [6]. The crack propagates to the right through 9 elements under constant load from an initial stationary position to the center of section A in Fig. 5. The same orientation of the crack with respect to the crystal is used in the propagating crack case as in the stationary crack case above. The yield surface for this orientation is repeated in Fig. 8 a for reference. As discussed in the previous section on
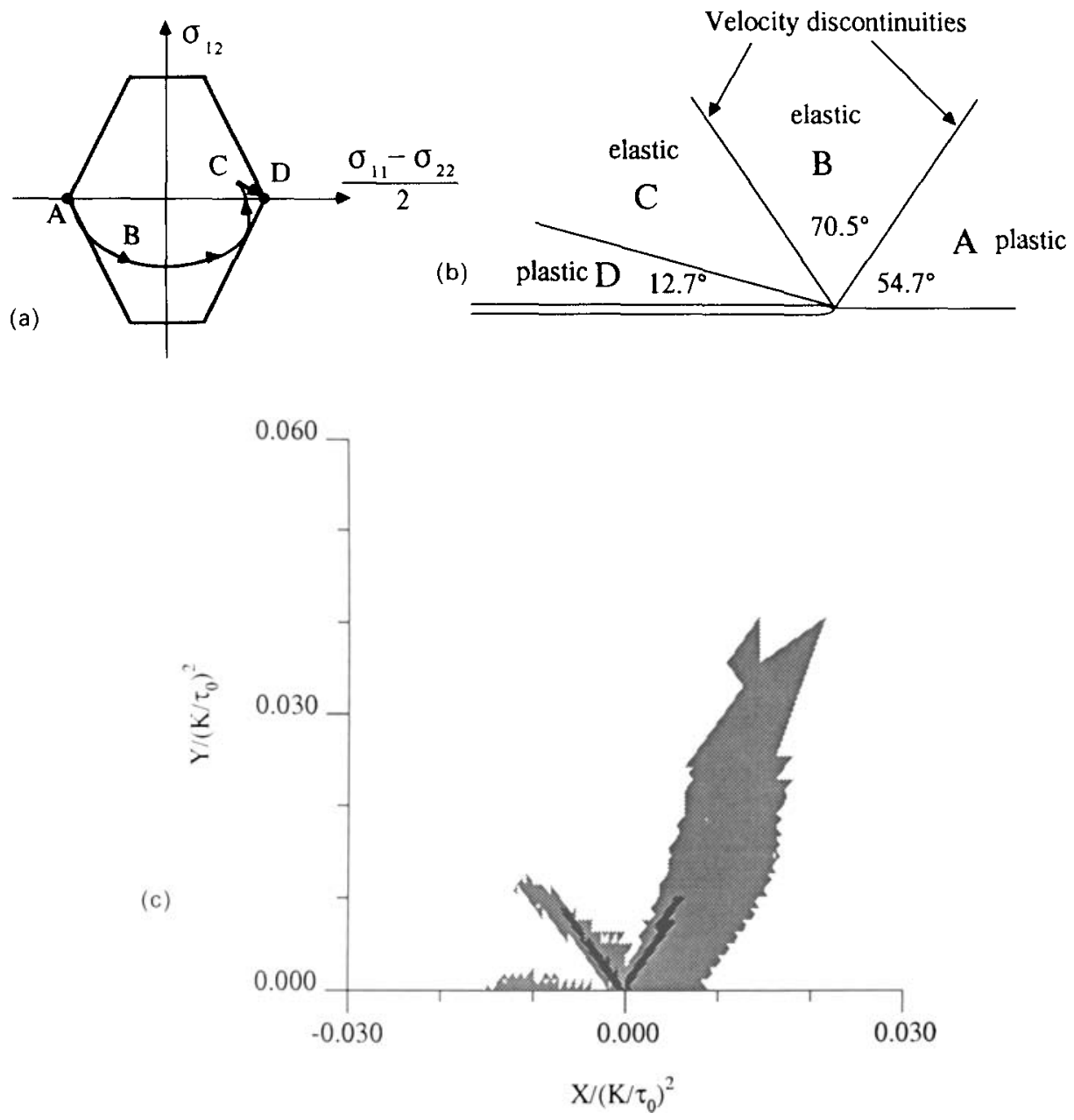

Fig. 8. Quasi-statically growing crack in ideally plastic crystal of Fig. 2: (a) Yield surface and stress trajectory. (b) Asymptotic structure of near tip field. (c) Finite element results showing plastic activity, as measured by increment of $\Gamma$, during one finite element step of crack growth. Grey zone corresponds essentially with plastically active zone during growth; black zone has more concentrated plastic straining. 
asymptotic solutions, neither stress nor displacement discontinuities may exist and if a sector is plastic it must border an elastic sector. The assembly of sectors for this orientation [2] is shown in Fig. 8b. For the upper half plane, the solution again consists of four sectors labeled A, B, C, and D. Sector A is a constant stress plastic sector and it is separated from an elastic sector $\mathrm{B}$ by a discontinuity in velocity at $54.7^{\circ}$. As indicated by the stress trajectory in Fig. $8 \mathrm{a}$, sector $\mathrm{B}$ involves an elastic unloading and reloading back to yield by the time a second velocity discontinuity is reached at $125.3^{\circ}$. Sector $\mathrm{C}$ is another elastic unloading and reloading sector. Finally, sector D is plastic.

The crack leaves behind a wake of plastic deformation which makes the quantity $\Gamma$ not as illustrative as it had been in the stationary crack case. The increment in $\Gamma /\left(\tau_{0} / G\right)$ $\left(=\Delta \Gamma /\left(\tau_{0} / G\right)\right.$ ) from just before the last node release to just after it, is shown in Fig. $8 \mathrm{c}$, which eliminates much of the accumulated plastic wake and shows only the regions which are plastically active during growth. Elements which sustained $\Delta \Gamma /\left(\tau_{0} / G\right)$ between 0.001 to 1 are shaded gray and those with increments greater than 1 are in shaded black. There are very striking similarities between the predicted asymptotic solution, Fig. 8b, and the finite element solution, Fig. 8c. Sector A is plastic and is bounded by an intense band of plastic deformation along the predicted velocity discontinuity. Sector B is relatively free of plastic deformation as predicted. At the second velocity discontinuity at $125.3^{\circ}$ where the predicted stress trajectory is tangent to the yield surface an intense band of plastic deformation is encountered. Subsequently, an elastic sector roughly similar in angular extent to sector $\mathrm{C}$ is seen in the finite element solution. Finally, elements along the crack faces are loading plastically. The deformed mesh after the crack growth has taken place is shown in Fig. 9a. It is interesting to note that while the crack was stationary (as load was increased) the solution was the same as in Fig. 7a, as shown by the distinct kink in the crack profile at the crack's initial position. However, once crack growth began and a displacement discontinuity was no longer allowed the crack profile was smooth. The increment in nodal displacements, again scaled up for visibility, from just before the final crack growth increment to just after is shown in Fig. 9b. Two rather distinct bands of discontinuity are seen close to the crack tip corresponding to the lines of the velocity discontinuities. The smooth change of direction of the increments directly above the crack clearly indicates that the discontinuity seen at $90^{\circ}$ in the stationary case is no longer present in the growing crack case.

\subsection{Center crack panel results}

A square, center cracked panel under plane strain conditions has been modeled from small-scale-yielding to general yielding in the finite element analyses of Asaro [6]. Three sections of the finite element mesh are shown in Fig. 10. Section A is imbedded in the dense part of section B which is in turn imbedded in the densest part of section C. Section C is then extended outwards in a similar pattern to slightly more than 20 times the size shown, with the outer boundaries of successive "rings" of elements forming two adjacent sides of a succession of squares. Symmetry reduces the analysis to just that of one quarter of the specimen, represented by section $C$ as extended to a large square with sides of dimension $b$. The total length of the crack is $2 \mathrm{a}$ with the ratio of $a / b$ equal to 0.01 . The ratio of the size of elements in section A to the size of the elements in the outermost mesh is approximately $2 \times 10^{-6}$. The crack tip is initially rounded as we can see from the mesh in section A. Stresses 
(a)
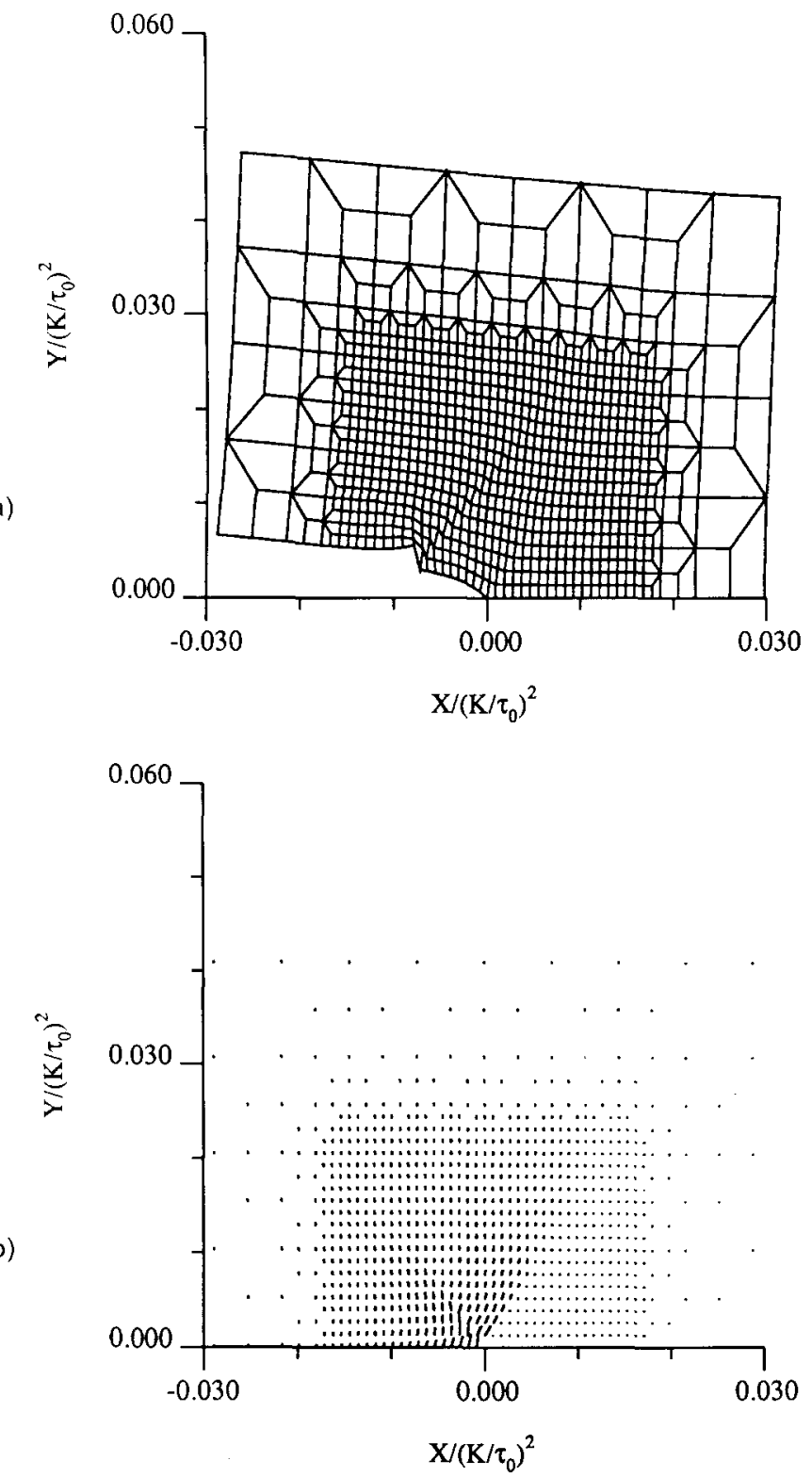

Fig. 9. (a) Deformed mesh (factor of 100 amplification) after several steps of crack growth at constant far-field stress intensity $K_{\mathrm{I}}$. (b) Line lengths proportional to displacement increments during crack growth over one finite element step of crack growth. Suggests that velocity discontinuities predicted by asymptotic analysis extend well into the plastic zone.

corresponding to uniform tension $\sigma^{\infty}$ perpendicular to the crack are applied to the outer boundary.

Finite rotations of both material and the crystal lattice are taken into account in this work. The computational procedures used in the finite element calculations follow the initial work of [14] and [18]. The elements used are the same cross triangle type [17] discussed in conjunction with the small scale-yielding analysis. The plasticity is modeled by a visco-plastic formulation with the slip rate on each system as given by (5) with $m=0.005$. A Taylor type 

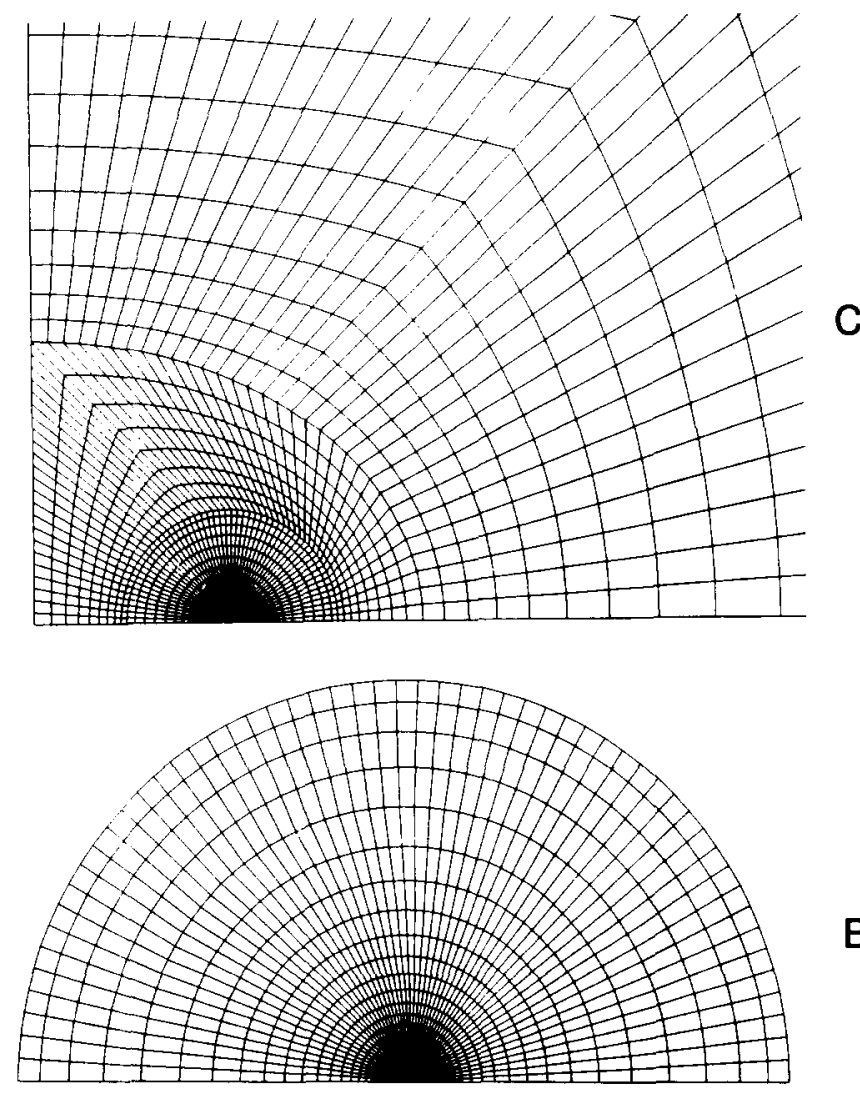

B

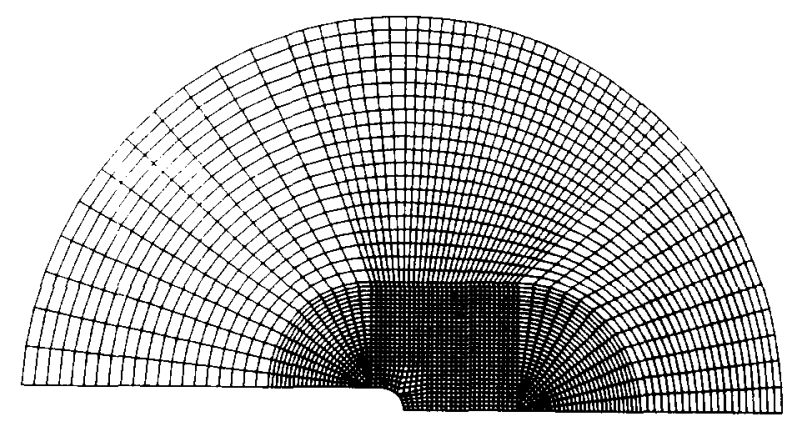

A

Fig. 10. Finite element mesh for analysis of tensile load of a panel containing a center crack with initially rounded tip. Used for crystal models with double slip, as in insets of Figs. 11(a) and (b). Strain hardening and arbitrary displacement gradients are included in the analysis.

of hardening of slip systems is included with $g^{(\alpha)}$ a function of $\Gamma=\Sigma_{\alpha}\left|\gamma^{(\alpha)}\right|$,

$$
g^{(\alpha)}=g_{0}[1+0.8 \tanh (11.1 \Gamma)]
$$

where $g_{0} / G=0.0026$.

The crystal is idealized by a planar double slip model for two orientations of the crack as shown by the insets of Fig. 11a and $11 \mathrm{~b}$. The first orientation corresponds to that in Fig. $2 \mathrm{~b}$ 

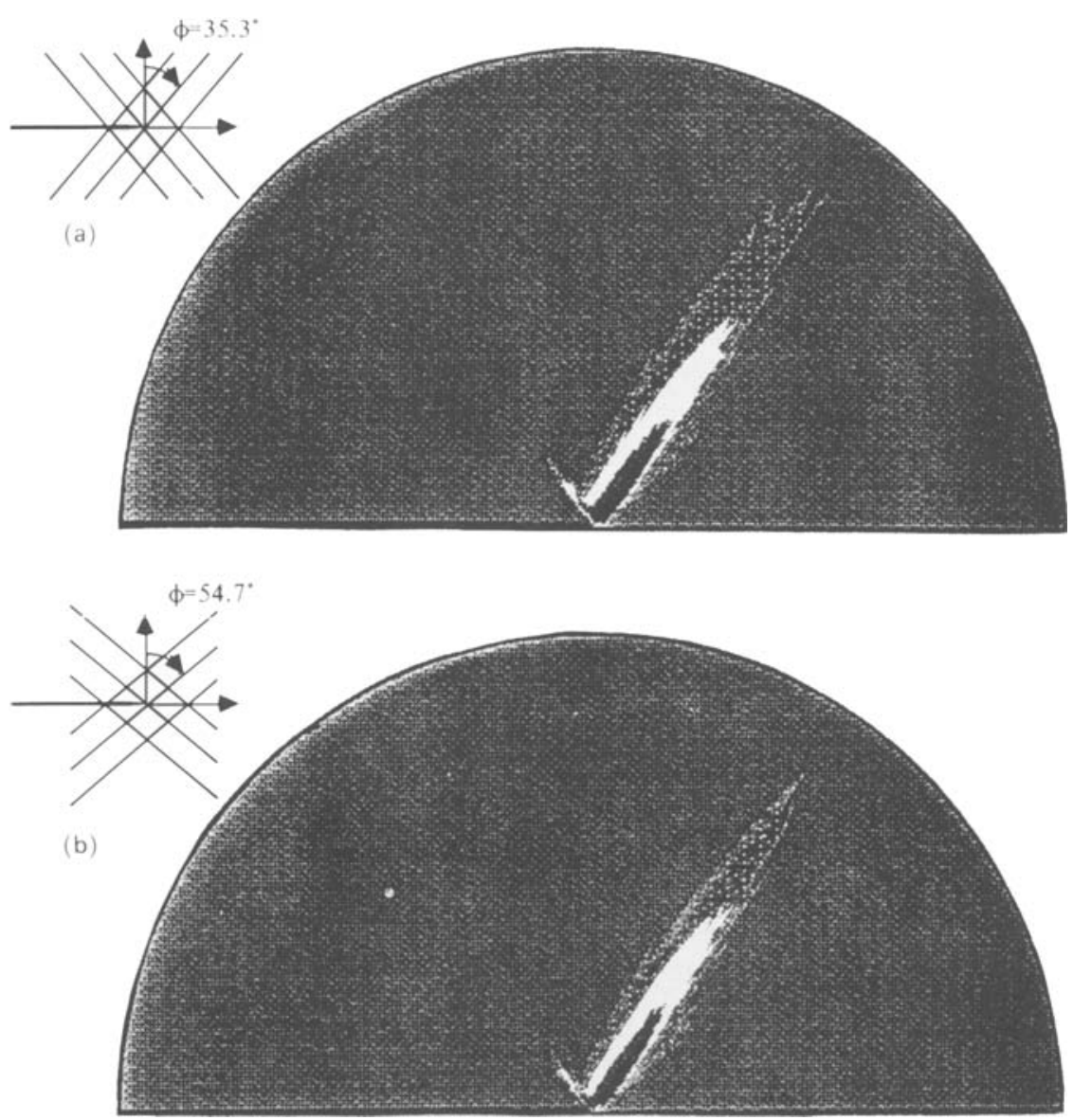

Fig. 11. Contours of equivalent shear strain $\Gamma$ near the tip; outermost is $\Gamma=0.005$, next is $\Gamma=0.01$ and innermost is $\Gamma=0.02$ : (a) For $35.3^{\circ}$ angle between slip planes and tensile direction; (b) For $54.7^{\circ}$ angle.

for a $\mathrm{fcc}$ crystal, i.e., a $\left(\begin{array}{lll}0 & 1 & 0\end{array}\right)$ crack growing in the [ $\left.\begin{array}{lll}1 & 0 & 1\end{array}\right]$ direction, when we neglect the slip plane traces shown as the dashed lines. It also corresponds to a $b c c$ crystal with the crack on the ( $\left.\begin{array}{lll}1 & 0 & 1\end{array}\right)$ plane growing in the $\left[\begin{array}{lll}0 & 1 & 0\end{array}\right]$ direction when we similarly neglect certain slip plane traces parallel to the crack. The angle between the $x_{2}$ axis and the traces of the slip direction $\mathrm{S}$, denoted by $\phi$, is equal to $35.3^{\circ}$ in this case. The second orientation has $\phi=54.7^{\circ}$. It corresponds, with neglect of certain systems as above, to a crack lying on the $\left(\begin{array}{ll}1 & 0\end{array}\right)$ plane growing in the direction $\left[\begin{array}{lll}0 & 1 & 0\end{array}\right]$ in a $\mathrm{fcc}$ crystal, or to a crack on the $\left(\begin{array}{lll}0 & 10) & 0\end{array}\right.$ plane growing in the [l 101 1] direction in a bcc crystal. See [2] for fuller discussion of yield surfaces in these cases. These orientations with only the slip systems indicated correspond to a diamond shaped yield surface, e.g., as in Figs. 6a and 8a but without the horizontal cut-offs. An identical diamond-shaped surface applies for the two cases shown in Fig. 11a and b. The f c c crack orientation coinciding with Fig. 11a is sometimes observed in fatigue studies on ductile $\mathrm{Cu}$ and $\mathrm{Al}$ crystals, whereas the bcc crack orientation noted to coincide with Fig. $11 \mathrm{~b}$ is a common cleavage crack orientation, e.g., in Fe-3\% Si crystals [16]. The term $g_{0}$ in (8) is the critical resolved shear stress in the $x_{1}, x_{2}$ plane of deformation for the double slip model. It corresponds to $g_{0}=(2 / \sqrt{3}) \tau_{0}$ for the f c c interretations and to $g_{0}=\tau_{0}$ for bcc [2]. 
Contours of constant plastic strain (as measured by the accumulated sum of the slips, $\left.\Gamma=\Sigma_{\alpha}\left|\gamma^{(x)}\right|\right)$ are shown in Figs. $11 \mathrm{a}$ and $11 \mathrm{~b}$ for the orientations $\phi=35.3^{\circ}$ and $\phi=54.7^{\circ}$ respectively. The outermost contour is for $\Gamma=0.005$, the next is for $\Gamma=0.01$ and the innermost for $\Gamma=0.02$. Away from the immediate near-tip region, the plastic deformation is confined to two discrete bands of deformation emanating from the crack tip. For a diamond shaped yield surface, the vertical $\left(\theta=90^{\circ}\right)$ discontinuity present in the small-scale yielding case (associated with the slip system represented by the dashed line in Fig. $2 b$ ) does not exist. The deformation in Fig. 11 a corresponds to shear bands with the line of discontinuity parallel to the slip direction $\mathbf{S}$. However, the deformation in Fig. 11b has the discontinuities aligned with the slip plane normal $\mathbf{N}$ which was discussed earlier as giving rise to kink bands and lattice rotation; see Fig. $3 \mathrm{~b}$ and $3 \mathrm{~d}$. Not until we are within two to three initial root radii of the initial blunted crack tip does the straining pattern deviate significantly from that predicted from the small-scale yielding perfectly sharp crack tip results, based on the "small displacement gradient" formulation. The solutions for the two cases in Fig. 11 are identical within that formulation, and the slight broadening of the strain contours in Fig. $11 \mathrm{~b}$, compared to those in Fig. 11a, reflects geometrical hardening due to the lattice rotation in the Fig. $11 \mathrm{~b}$ case.

The maximum extent of the plastic zone $r_{p}$ varies nearly linearly with $a\left(\sigma^{\infty} / g_{0}\right)^{2} \propto K_{1}^{2}$ for values of $r_{p} / a \leqslant 0.018$, such that $r_{p} \approx 0.14 a\left(\sigma^{\infty} / g_{0}\right)^{2}$. (The corresponding numerical factor is $\approx 0.15$ in the ideally plastic fcc solution of Hawk ([6] Fig. $6 \mathrm{c}$ ), and both results are close to the factor 0.16 based on the approximate ([19] Eqn. (37b)) model for yield on a pair of inclined shear planes at a crack tip, when the angle of those planes is taken as $55^{\circ}$ with the cracking direction.) For larger plastic zone sizes in the Asaro solution, departures from such linearity occur indicating a departure from self-similar growth of the plastic zone, i.e., a violation of small-scale yielding, presumably explainable over some range of $\sigma^{\infty}$ prior to full plasticity in terms of the nonsingular, crack-parallel stress $\left(=-\sigma^{\infty}\right)$ of the elastic crack tip field (e.g., [20]).

Lattice rotations from the initial orientations, caused by plastic deformation at the crack tip, are shown in Figs. 12a and 12b. These show the deformed orientations of the traces of one of the slip plane families in the double-slip model. For the case with $\phi=35.3^{\circ}$, Fig. $12 \mathrm{a}$ indicates that although quite large rotations occur at the crack tip itself, very little reorientation of the lattice occurred in the shear band regions. This is compatible with flow parallel to slip plane traces as in Fig. 3a. In contrast to this, Fig. 12b indicates, for the case where $\phi=54.7^{\circ}$, rather discrete bands of lattice reorientation occur that more-or-less coincide with the bands of concentrated straining of Fig. 11b. By sighting along the slip plane traces in this latter case, one may observe a zone of kinking shear, as in Fig. 3b, extending out from the crack tip. This is further illustrated in Fig. $12 \mathrm{c}$, also for the $\phi=54.7^{\circ}$ case, in which contours of constant lattice rotation are shown. The outermost contour corresponds to a $2^{\circ}$ rotation, and it protrudes out from the crack tip in the directions of the bands of concentrated straining in Fig. $11 \mathrm{~b}$; no such outward protrusion of regions of significant lattice rotation occurs in the $\phi=35.3^{\circ}$ case. By comparing Figs. $12 \mathrm{a}$ and b, it is seen that although the overall plastic zone sizes differ little in the two cases, the patterns of large deformation in crack tip opening are quite different and seem to be dominated by the geometrical hardening and softening from lattice rotations. The results also show the development of features resembling "high-angle boundaries", or at least narrow transition zones between regions of significantly different lattice rotation, in the near tip region. 
(a)

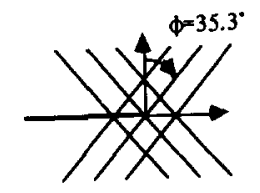

(b)
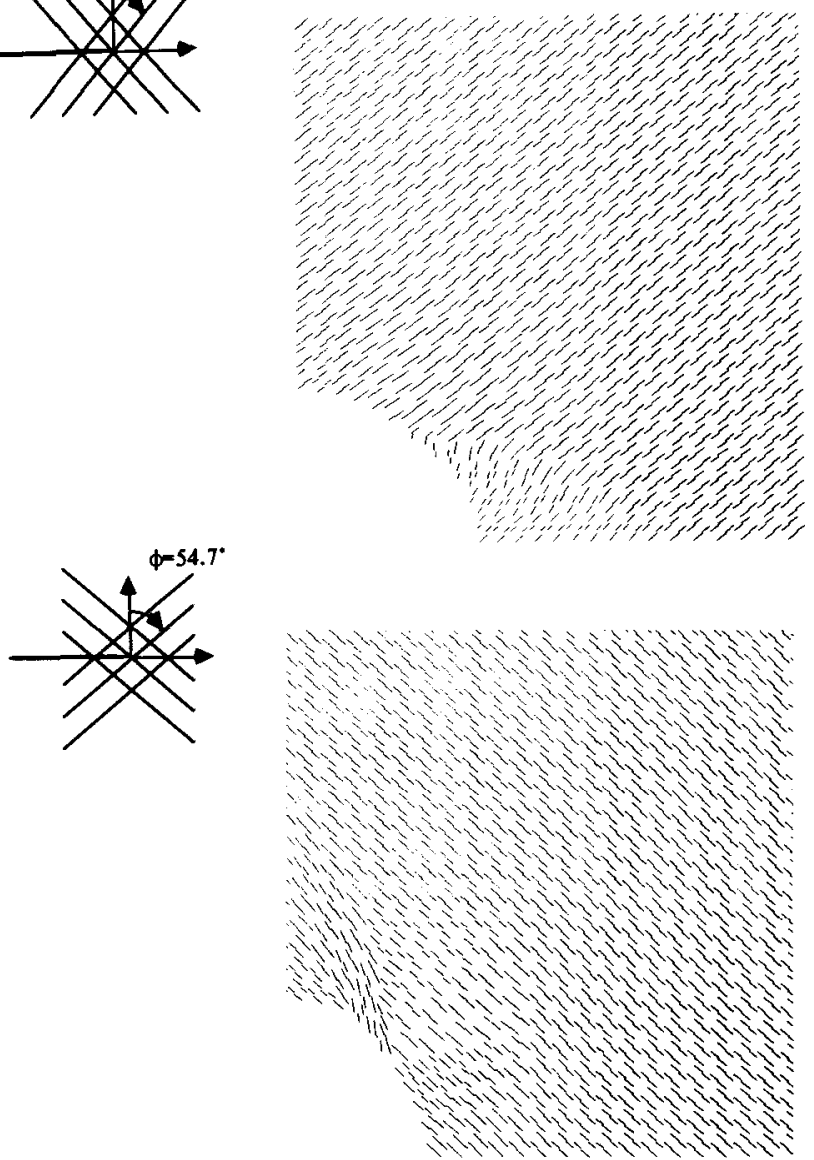

(c)

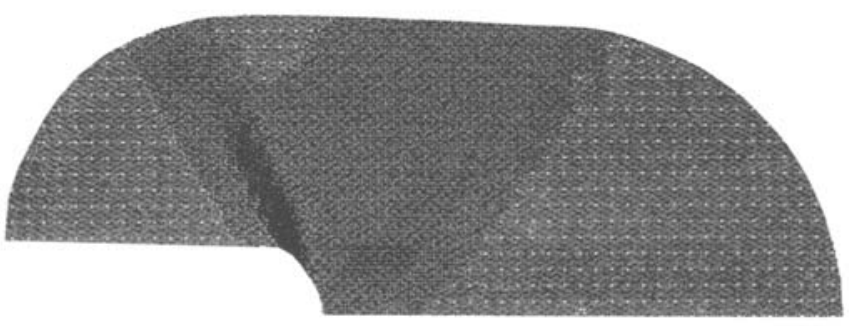

$-7.5000$

$-10.0000$

$-15.0000$

$-20.0000$

Fig. 12. Rotated orientations of slip plane traces (from one of the two slip systems in the double-slip model) near the crack tip: (a) For $35.3^{\circ}$ angle between planes and tensile direction; shows little evidence of lattice rotation except near blunting tip. (b) For $54.7^{\circ}$ angle; shows significant lattice rotation as in the kinking shear zone of Fig. 3(b). (c) Also for $54.7^{\circ}$ angle; shows contours of constant lattice rotation near the tip, of which the outermost corresponds to $2^{\circ}$ clockwise. 


\section{Conclusion}

The analytical and numerical investigations into the near-crack-tip fields of ductile crystals have been shown to be consistent. The results show that for ideally plastic crystals obeying the Schmid rule, the near-crack-tip deformation fields are characterized by discontinuities in either displacement (stationary crack) or velocity (quasi-statically growing crack). These discontinuities are either parallel or normal to crystallographic planes on which slip can take place (i.e. by the motion of dislocations) for the $f c c$ and $b c c$ orientations considered. The state of stress is constant within angular sectors bounded by these discontinuities for material at yield.

The asymptotic methods developed [1,2] provide a general way of constructing such fields around the crack once the yield surface has been determined for the particular crystal and orientation of the crack. In the case of a mode III crack $[1,3]$ a more complete analysis is possible of stationary cracks (exact full analysis) and quasi-static crack growth (asymptotic plus approximate analysis) and even dynamic crack growth (asymptotic). The general features seen in the mode III cases carry over to the physically more interesting mode I plane strain asymptotic analyses [2].

The numerical investigation of Hawk [6] by detailed finite element analyses provided confirmation that the features seen in the mode I asymptotic analyses (stationary and quasi-static growth) are valid over a finite size region. The finite element analysis of Asaro [6] of a center crack with initially rounded tip in a tension panel from small-scale yielding to general yielding, including hardening and full account of arbitrary displacement gradients, shows the effects of lattice rotation and the limits of small-scale yielding. However, the overall feature of concentrated deformation along the predicted discontinuities is retained throughout.

Finally, the analyses summarized here may provide some insight into why certain crystals undergo ductile fracture while others are brittle. One possible factor is the structure of the dislocations necessary to produce the predicted slip patterns which in one case involves a shear band parallel to the active slip system and in another involves a band perpendicular to the active system that deforms by kinking shear. In the parallel shear case it is possible for these dislocations to be generated at the crack tip and swept out along the slip planes while in the kinking case it is necessary to have present, and to activate, internal sources to generate the necessary dislocation dipoles. The finite strain pattern associated with large ductile opening at the crack tip is also significantly different in the two cases.

\section{Acknowledgements}

J.R. Rice and D.E. Hawk acknowledge the support of the Office of Naval Research under contract N00014-85-K-0045 to Harvard University. The computations of D.E. Hawk were carried out at the John von Neumann Center, Princeton N.J., under grant NAC-519 supported by the U.S. National Science Foundation. R.J. Asaro also acknowledges the support of the Office of Naval Research under contract N00014-88-K-0119 to Brown University. His computations were done during a June 1987 visit to Sandia National Laboratory, Livermore, Calif. 


\section{References}

1. J.R. Rice and R. Nikolic, Jounal of the Mechanic's and Phrsics of Solids 33 (1985) 595-622.

2. J.R. Rice, Mechanics of Materials 6 (1987) 317-335.

3. R. Nikolic and J.R. Rice, Mechanics of Materials 7 (1988) 163-173.

4. J.R. Rice and M. Saeedvafa, Joumal of the Mechanic's and Physics of Solids 36 (1988) 189-214.

5. M. Saeedvafa and J.R. Rice, Journal of the Mechanics and Physics of Solids (1989) in press.

6. D.E. Hawk and R.J. Asaro, work in progresss on finite element analysis of cracked eleastic-plastic crystals (1988).

7. G.I. Taylor and C.F. Elam, Proceedings of the Royal Socicty of London A 102 (1923) 643.

8. G.I. Taylor and C.F. Elam, Proceedings of the Roval Society of London A 108 (1925) 28.

9. G.I. Taylor, in Stephen Timoshenko 60th Anniversary Volume, Macmillan, New York (1938) 218-224.

10. R.J. Asaro, in Advances in Applied Mechanics, J.W. Hutchinson (ed.), 23 (1983) 1-115.

11. J.R. Rice, Journal of the Mechanics and Physics of Solids 21 (1973) 63-74.

12. J.W. Hutchinson, Proceedings of the Royal Society of London A350 (1976) 101-127.

13. J. Pan and J.R. Rice, International Journal of Solids and Strutures 19 (1983) 973-987.

14. D. Pierce, R.J. Asaro, and A. Needleman, Acta Metallurgica 31 (1983) 1951-1976.

15. W.J. Drugan and J.R. Rice, in Mechanics of Material Behavior, G.J. Dvorak and R.T. Shield (eds.), Elsevier, Amsterdam (1984) 59-73.

16. A.S. Tetelman and W.D. Robertson, Acta Metallurgica 11 (1963) 415-426.

17. J.C. Nagtegaal, D.M. Parks, and J.R. Rice, Computer Methods in Applied Mechanics and Engineering 4 (1974) 153-177.

18. R.J. Asaro and A. Needleman, Acta Metallurgica 33 (1985) 923-953.

19. J.R. Rice, in Fatigue Crack Propagation, ASTM STP 415 (1967) 247-309.

20. J.R. Rice, Journal of the Mechanics and Physics of Solids 22 (1974) 17-26. 